\title{
Boosting the boost: the effect of tidal stripping on the subhalo luminosity.
}

\author{
Richard Bartels* \\ University of Amsterdam \\ E-mail: r.t.bartels@uva.nl
}

\section{Shin'ichiro Ando}

University of Amsterdam

\begin{abstract}
In the paradigm of $\Lambda \mathrm{CDM}$, structures form hierarchically, implying that large structures contain smaller substructures. These so-called subhalos can enhance the dark matter annihilation signal that one expects to see from a given host halo, the effect of which is called the boost factor. In the literature this boost factor is typically calculated assuming a density profile for the substructure, or analogously a concentration-mass relation, corresponding to that of field halos. However, since subhalos accreted in a gravitational potential of their host loose mass through tidal stripping and dynamical friction, they have a quite characteristic density profile, different from that of the field halos of the same mass. In this work we attempt to quantify the effect of tidal stripping on the boost factor. We find that the boost factor increases by a factor few for host halos ranging from sub-galaxy to cluster masses.
\end{abstract}

The 34th International Cosmic Ray Conference

30 July- 6 August, 2015

The Hague, The Netherlands

\footnotetext{
${ }^{*}$ Speaker.
} 


\section{Introduction}

In the paradigm of cold dark matter it is believed that small dark matter (DM) structures form first, merging into larger ones. This is known as hierarchical structure formation and it leads to a clumpy distribution of DM inside halos. In addition, many particle physics models of DM allow for the possibility that DM can self-annihilate into high-energy gamma rays, making gamma-rays a promising probe to search for non-gravitational interactions of DM. Self-annihilation scales with the DM density squared. Therefore, if indeed DM halos are clumpy and a substantial fraction of the DM is locked in substructure, the annihilation signal should be enhanced compared to having a smooth DM halo only. This enhancement due to substructures is known as the boost factor. Including this boost factor, the total luminosity of a DM halo of mass $M(L(M))$ can be written as [1]

$$
\begin{aligned}
L(M) & =\left[1+B_{\mathrm{sh}}(M)\right] L_{\mathrm{host}}(M), \\
B_{\mathrm{sh}}(M) & =\frac{1}{L_{\mathrm{host}}(M)} \int \mathrm{d} m \frac{\mathrm{d} N}{\mathrm{~d} m} L_{\mathrm{sh}}(m)\left[1+B_{\mathrm{ssh}}(m)\right],
\end{aligned}
$$

here $L_{\text {host }}(M)$ is the luminosity of the halo its smooth DM component, $B_{\mathrm{sh}}$ is the boost factor due to substructure, $L_{\mathrm{sh}}(m)$ the luminosity of the smooth component of a mass $m$ subhalo, $\mathrm{d} N / \mathrm{d} m$ the subhalo mass function, and $B_{\text {ssh }}$ the boost due to sub-substructure. This latter component is usually believed to be small and ignored. Note that with this definition of boost a boost factor of $B=0$ means no boost, and a boost factor of $B=1$ means the expected self-annihilation signal is twice as strong compared to the smooth halo only.

The boost factor is sensitive to two things. First, it is highly sensitive to the subhalo mass function, $\mathrm{d} N / \mathrm{d} m \propto m^{-\alpha}$. From numerical simulations, the slope is found to be in the range $\alpha \in$ $[1.9,2][2,3]$. The steeper the slope, the more dominated the boost is by the smallest substructures and typically the larger the boost will be . Moreover, the boost is directly proportional to the normalisation, which is usually set by matching the subhalo abundance in the high-mass tail of the mass function to the results of simulations. Secondly, the boost is sensitive to the subhalo luminosity. Assuming a truncated NFW profile for the subhalos, the subhalo luminosity can be written as $L_{\mathrm{sh}}(m) \propto \rho_{\mathrm{s}}^{2} r_{\mathrm{s}}^{3}\left[1-1 /\left(1+c_{\mathrm{t}}\right)^{3}\right]$ [4]. Here $\rho_{\mathrm{s}}$ and $r_{\mathrm{s}}$ are the scale density and scale radius of the NFW profile. The concentration is given by $c_{\mathrm{t}} \equiv r_{\mathrm{t}} / r_{\mathrm{s}}$, with $r_{\mathrm{t}}$ the tidal radius. However, typically in the literature, when calculating the boost this luminosity is taken to be the same as that of field halos of the same mass. But field halos tend to be less concentrated and consequently less luminous. For subhalos, on the other hand, tidal stripping will remove the outer less dense part of the halo, leaving behind a more concentrated object. In this work, we attempt to estimate the magnitude of this effect and make a more realistic estimate of the boost by taking into account tidal stripping.

Throughout this paper/letter we adopt the WMAP5 cosmological parameters [5]. We take capital $M$ to refer to the host halo mass and lower-case $m$ to refer to subhalo mass, unless mentioned otherwise. 


\section{Methods}

For all halos we assume an NFW profile. Subhalos are approximated by a truncated NFW

$$
\rho(r)= \begin{cases}\rho_{\mathrm{NFW}}(r) & r \leq r_{\mathrm{t}} \\ 0 & r_{\mathrm{t}}<r .\end{cases}
$$

We assume $\rho_{\mathrm{s}}$ and $r_{\mathrm{s}}$ to remain unchanged throughout the evolution of the subhalo. At the time of accretion of the subhalo by the host (at redshift $z_{\mathrm{a}}$ ), we take the progenitor to have mass $m_{\mathrm{a}}$. At this point we fix the aforementioned scale radius and density by matching the concentration $c_{200}=r_{200} / r_{\mathrm{s}}$ to the concentration-mass-redshift relation of [6]. Here $r_{200}$ refers to the radius within which the mean density of the progenitor is 200 times that of the critical density at $z_{\mathrm{a}}$.

Next, we evolve the subhalo down to redshift 0 . For this purpose, we apply the semi-analytical model from [7]. This model provides an orbit-averaged mass loss rate for subhalos, allowing us to estimate the expected mass of a halo at a given redshift $z, m\left(z, z_{\mathrm{a}}, m_{\mathrm{a}}, M_{0}\right)$. We assume this relation to model to hold down to $m \sim 10^{-6} \mathrm{M}_{\odot} . M_{0}$ is the mass of the host halo today $(z=0)$. For the host halo mass accretion history we apply the analytical model by [8]. This model derived from extended Press-Schechter theory (EPS) [9], gives a mean evolution for a host of final mass $M_{0}$, $M\left(z, M_{0}\right)$.

Finally, accretion times and masses are distributed following [10]. They provide an analytical EPS model for $\mathrm{d} N / \mathrm{d} \ln m_{\mathrm{a}} / \mathrm{d} \ln \left(1+z_{\mathrm{a}}\right)$.

With these inputs we can determine the evolved subhalo mass function in terms of final subhalo mass and final concentration, $\left(m_{0}, c_{\mathrm{t}}\right)$. This is proportional to the probability of finding a given mass subhalo with a given concentration,

$$
\begin{aligned}
P\left(m_{0}, c_{\mathrm{t}} \mid M_{0}\right) & \propto \frac{\mathrm{d}^{2} N}{\mathrm{~d} \ln m_{0} \mathrm{~d} c_{\mathrm{t}}} \\
& =\frac{\mathrm{d}^{2} N}{\mathrm{~d} \ln m_{a} \mathrm{~d} \ln \left(1+z_{\mathrm{a}}\right)}\left|\frac{\partial\left(\ln m_{\mathrm{a}}, \ln \left(1+z_{\mathrm{a}}\right)\right)}{\partial\left(\ln m_{0}, c_{\mathrm{t}}\right)}\right| .
\end{aligned}
$$

\section{Results}

For host halos in the range $10^{6} \mathrm{M}_{\odot}$ to $10^{16} \mathrm{M}_{\odot}$ we find the slope of the evolved subhalo mass function to be in the range $1.91-1.94$ and the mass fraction in subhalos down to $m \sim 10^{-6} \mathrm{M}_{\odot}$ to be $5-25 \%$. In agreement with the results from numerical simulations $[2,3]$.

Using Eq. 2.2 the average luminosity of a mass $m$ subhalo can be calculated as follows

$$
L_{\mathrm{sh}}(m)=\int_{1}^{c_{\max }} L\left(m_{0}=m, c_{\mathrm{t}}\right) P\left(c_{\mathrm{t}} \mid m_{0}=m\right) \mathrm{d} c_{\mathrm{t}} .
$$

In Fig. 1 we show the ratio of the boost for different host halo masses assuming subhaloss are stripped and have a luminosity as given by Eq. 3.1, compared to the boost under the assumption that subhalos have a luminosity which is identical to that of field halos. In the latter case the concentrations are taken from [6]. We adopt four fiducial models to show the dependence on the subhalo-mass function. Solid lines refer to a minimum halo mass of $m \sim 10^{-6} \mathrm{M}_{\odot}$ and dashed lines to one of $m \sim 10^{4} \mathrm{M}_{\odot}$. Also we adopt slopes of both 1.9 and 2.0. As can be seen, irrespective of 
the subhalo mass function, the boost is typically a factor 2-3 larger when taking into account the effects of tidal stripping.

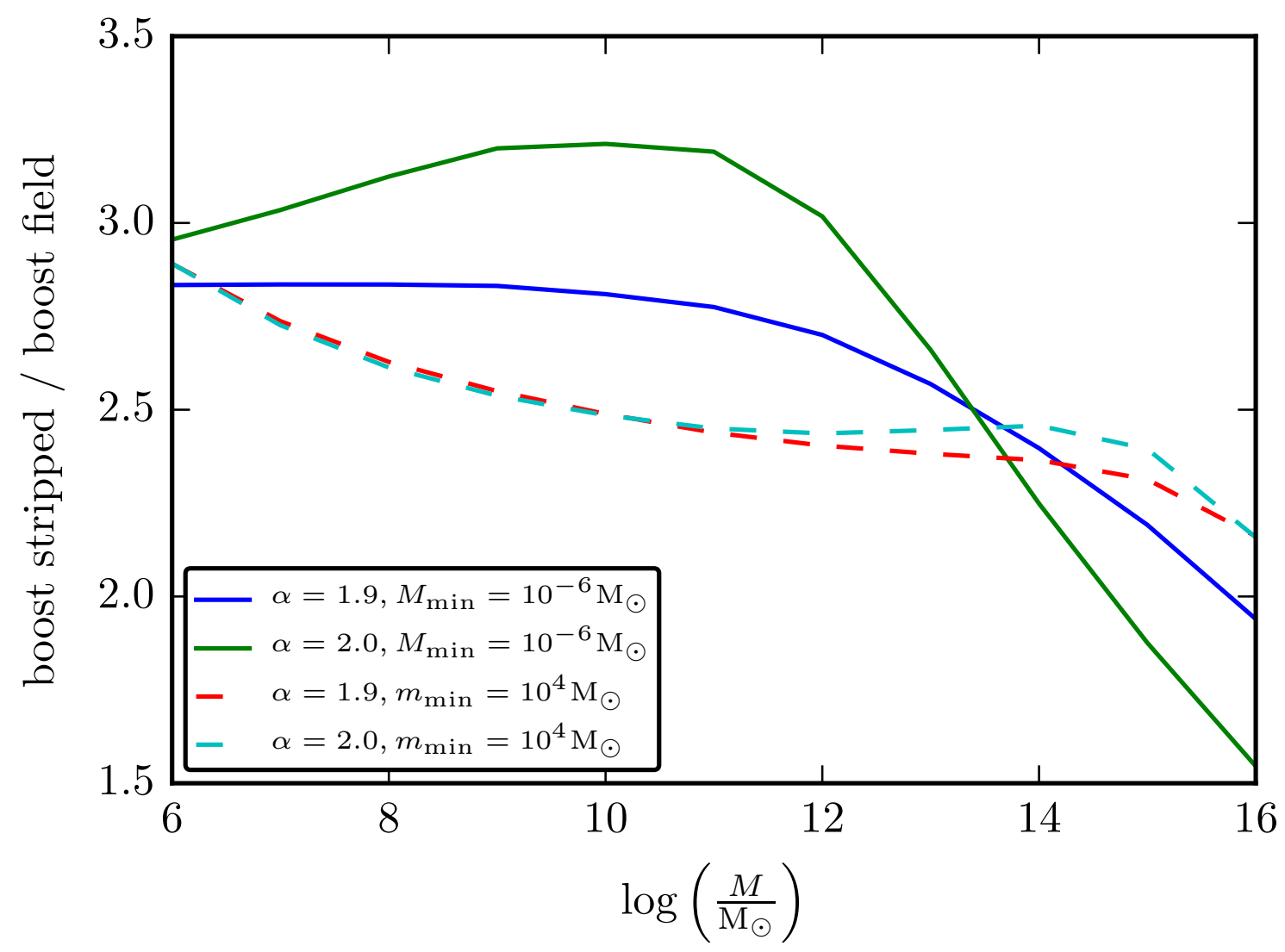

Figure 1: The ratio of the boost assuming the subhalos are stripped over that where the subhalos have the same luminosity as field halos for different host halo masses. We adopt four fiducial models, with minimum subhalo masses of $m_{\min } \in\left[10^{-6} \mathrm{M}_{\odot}, 10^{4} \mathrm{M}_{\odot}\right]$ and slopes of the subhalo mass function of $\alpha \in[1.9,2]$.

In Fig. 2 we show the overall boost factor when tidal stripping is taken into account for different subhalo mass functions. The solid line corresponds to a self-consistent modelling, where we apply the subhalo mass function that comes out of our analysis. The dotted lines use the same subhalo mass functions as [11]. Note that apart from the tidal stripping, these results also differ from those in [11] because we assumed the concentration-mass relation of [6] rather than from [11]. In addition, we show the expected boost for Milky-way like dwarf satellites using the self-consistent modelling. These dwarfs are expected to have a smooth component that is brighter than similar mass field halo objects, since they are stripped themselves. Moreover, they also loose part of their substructure due to tidal stripping, resulting in a boost that is smaller compared to the boost of field halo objects by about an order of magnitude.

\section{Conclusion}

We studied the concentration and luminosity of dark matter subhalos taking into account tidal 


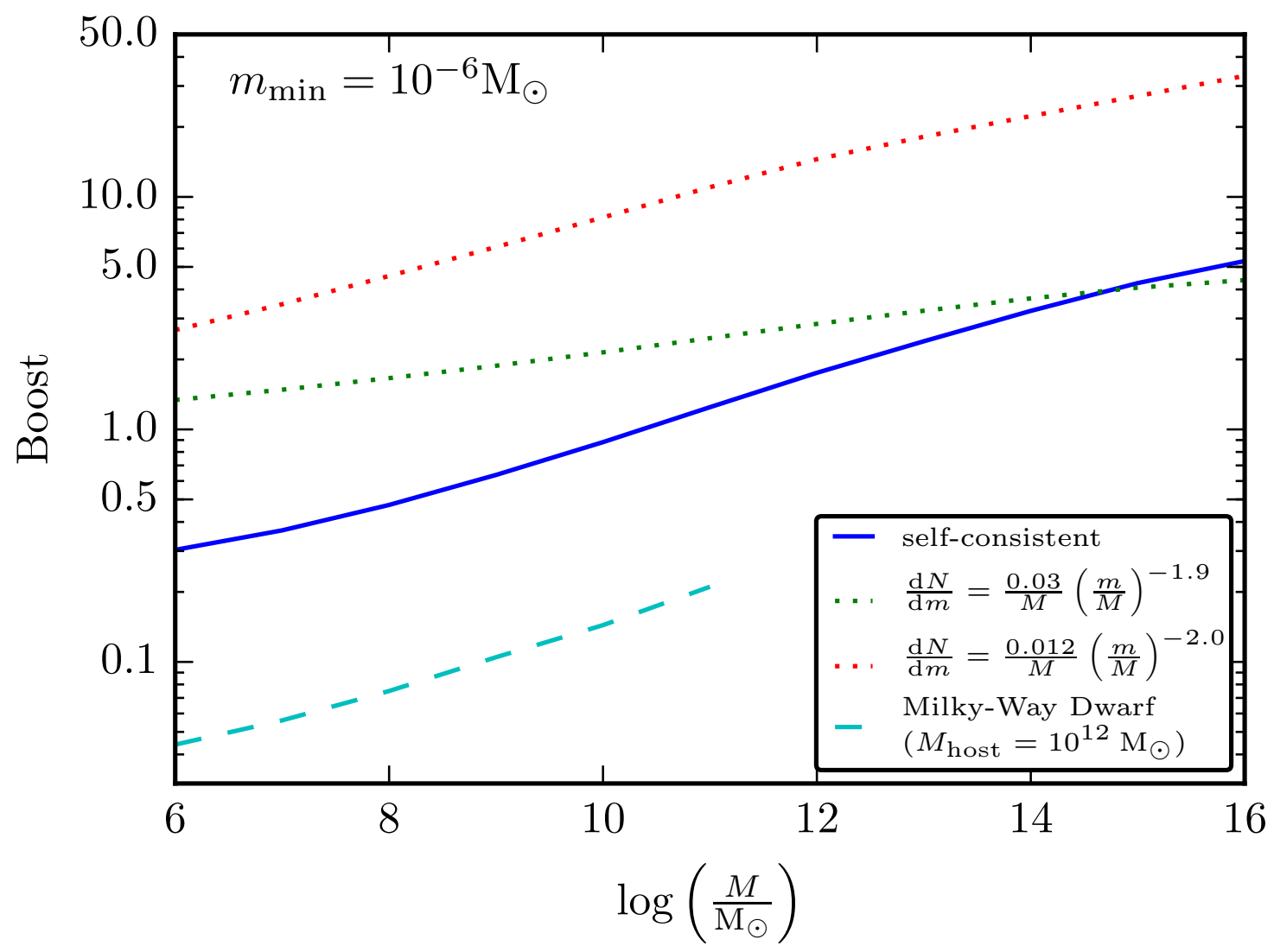

Figure 2: The boost factors for halos of different size when tidal stripping is taken into account for different subhalo mass functions. The solid line is corresponds to a self-consistent modelling of the subhalo luminosities and the subhalo mass function. The dotted lines assume the same subhalo mass function as in [11]. In addition, we show the expected boost for dwarf satellites of the Milky way. This is smaller by a factor $\mathscr{O}(10)$ since they themselves will be more luminous due to stripping and since they will have lost about two-thirds of their subhalos.

stripping. We find that subhalos are significantly brighter, by about a factor of 2-3, than their halos of the same mass in the field. Taking this into account when calculating the boost factor leads to a similar increase in the boost compared to traditional results in the literature.

\section{References}

[1] L. E. Strigari, S. M. Koushiappas, J. S. Bullock, and M. Kaplinghat, Precise constraints on the dark matter content of Milky Way dwarf galaxies for gamma-ray experiments, Phys.Rev. D75 (2007) 083526, [astro-ph/0611925].

[2] J. Diemand, M. Kuhlen, and P. Madau, Dark matter substructure and gamma-ray annihilation in the Milky Way halo, Astrophys. J. 657 (2007) 262-270, [a stro-ph/ 0611370 ].

[3] V. Springel, J. Wang, M. Vogelsberger, A. Ludlow, A. Jenkins, et al., The Aquarius Project: the subhalos of galactic halos, Mon.Not.Roy.Astron.Soc. 391 (2008) 1685-1711, [arXiv : 0809.0898 ]. 
[4] J. F. Navarro, C. S. Frenk, and S. D. White, The Structure of cold dark matter halos, Astrophys.J. 462 (1996) 563-575, [astro-ph/9508025].

[5] WMAP Collaboration Collaboration, E. Komatsu et al., Five-Year Wilkinson Microwave Anisotropy Probe (WMAP) Observations: Cosmological Interpretation, Astrophys.J.Suppl. 180 (2009) 330-376, [arXiv:0803.0547].

[6] C. A. Correa, J. S. B. Wyithe, J. Schaye, and A. R. Duffy, The accretion history of dark matter halos III: A physical model for the concentration-mass relation, arXiv: 1502.0039.

[7] F. Jiang and F. C. v. d. Bosch, Statistics of Dark Matter Substructure: I. Model and Universal Fitting Functions, arXiv:1403.6827.

[8] C. Correa, S. Wyithe, J. Schaye, and A. Duffy, The accretion history of dark matter halos I: The physical origin of the universal function, arXiv:1409.5228.

[9] W. H. Press and P. Schechter, Formation of galaxies and clusters of galaxies by selfsimilar gravitational condensation, Astrophys.J. 187 (1974) 425-438.

[10] X. Yang, H. Mo, Y. Zhang, and F. C. d. Bosch, An analytical model for the accretion of dark matter subhalos, Astrophys.J. 741 (2011) 13, [arXiv:1104.1757].

[11] M. A. Sanchez-Conde and F. Prada, The flattening of the concentration-mass relation towards low halo masses and its implications for the annihilation signal boost, Mon.Not.Roy.Astron.Soc. 442 (2014) 2271, [arXiv:1312.1729]. 